Vietnam Journal of Mechanics, VAST, Vol.38, No. 4 (2016), pp. 223 - 238

DOI:10.15625/0866-7136/6235

\title{
FREQUENCY RESPONSE OF A BEAM-LIKE STRUCTURE TO MOVING HARMONIC FORCES
}

\author{
Nguyen Tien Khiem ${ }^{1, *}$, Phi Thi Hang ${ }^{2}$ \\ ${ }^{1}$ Institute of Mechanics, VAST, Hanoi, Vietnam \\ ${ }^{2}$ Electric Power University, Hanoi, Vietnam \\ *E-mail: ntkhiem@imech.ac.vn \\ Received May 23, 2015
}

\begin{abstract}
The spectral approach is employed for spectral analysis of a beam subjected to an arbitrary force traveling along the beam with constant speed. First, an expression for exact frequency response of a beam subjected to moving arbitrary force and general boundary conditions has been constructed. The obtained frequency domain response allows straightforwardly exhibiting response vibration components governed by different frequencies such as the natural, loading and driving ones and their interaction. This provides also alternative insight to the cancellation of response at natural frequency. The theoretical development is illustrated and validated by numerical examination on a simply supported beam under moving harmonic forces.
\end{abstract}

Keywords: Moving force, frequency response, spectral analysis, resonance, cancellation.

\section{INTRODUCTION}

The moving load problem for a long time has attracted attention of researchers and engineers in the field of structural engineering and it is so far an actual topic in dynamics of structures. The fundamentals of the problem were formulated in [1-5] and intensively studied in the widespread literature, for example, the references [6-11]. In the most of the studies, the problem has been investigated by using the analytical method based mainly on the superposition principle. Latter, the FEM $[12,13]$ and, recently, the spectral approach [14-16] has been developed for dynamic analysis of beams subjected to various types of moving load. However, the moving load problem was mostly solved in the time domain even when the spectral element method has been employed. There are very few works devoted to study the moving load problem in the frequency domain and it is subject of present paper.

The most important issue in the dynamic analysis of structures subjected to moving load is to evaluate the dynamic amplification factor (DAF) defined by structure's

(C) 2016 Vietnam Academy of Science and Technology 
response maximum in dependence on the load roving speed. Savin [17] obtained a socalled dynamic amplification coefficient defined as the maximum of modal coordinates with respect to time and depicted it versus a frequency factor. Pesterev et al. [18] proved that there exists a function describing the dependence of the global maximum (with respect to both the spatial and time variables) on travelling speed of constant force. These functions referred to as the modal response functions (MRF) were constructed for simply supported and clamped beams by using the first mode approximation of time domain response. Both the latter studies proposed to divide the time history response into two components: forced and free vibration modes by the time instant as the load is passed over the beam. This characterization of time history response may break down its natural vibration mode that takes part into both the vibration components. To avoid the concern an alternative approach that characterizes the response by components of different frequencies can be used as done by Yang and Lin [19]. The latter authors demonstrated that bridge response in time domain consists mainly of natural and driving frequency components (the vehicle frequency component as forced vibration mode was omitted due to approximation). It was reported in [19] that speed of vehicle is associated only with the peaks of lower frequencies and bridge frequency is most dominated in the response of the vehicle. The dynamic response representation has been incorporated by the forced vibration mode in $[20,21]$ where bridge response to moving harmonic force is comprehensively investigated in time domain. In the Ref. [20], the influence of the parameters such as load frequency and phase on the maximum amplification at mid-span has been studied. The so-called transient frequency response was constructed and used by the authors of Ref. [21] to study vibration amplitude at the natural resonance when load frequency equals to the natural frequencies of beam. Note that the fundamental resonant vibration of a girder bridge under high speed trains was thoroughly studied by $\mathrm{Li}$ and $\mathrm{Su}$ [3]. It is worth to recall herein an early study [22] where a curious phenomenon that describes cancellation of natural frequency response of a simple beam subjected to moving load was revealed. Though the cancellation condition was derived in [22] from the first mode approximation, it is valid also for total response at the fundamental frequency. This phenomenon of the cancellation was studied further for beam with elastic bearings [23, 24] and non-prismatic beam [25]. Although the cancellation speeds were calculated for the first and second modes in [24] but actual cancellation speeds of total response have just been determined from the first mode.

All the aforementioned studies were based on the time domain solution that is obtained by using either the superposition or finite element methods that work well only in the case if contribution of higher modes could be negligible. The present paper is devoted to develop the approach proposed in [16] for spectral analysis of response of a beam-like structure subjected to arbitrary force traveling on the structure with constant speed. The most important difference of this study in comparison with the early published works consists of exact solution obtained for frequency response to moving arbitrary force. The developed herein approach enables not only to study the total dynamic response in arbitrary frequency range but also to develop a new characterization of the dynamic response with different frequencies. Besides, the cancellation condition that was noticed in $[17,18]$ and studied in [22-25] based on the various approximations to the time history response 
can be easily derived herein for total frequency response and this phenomenon is thoroughly investigated for harmonic loads. Without loss of generality, numerical analysis is accomplished for simply supported beam subject to combined harmonic loads. The problem for beam with other boundary conditions and more complete moving loads can be similarly solved by using the spectral approach developed above.

\section{FREQUENCY RESPONSE OF BEAM TO MOVING ARBITRARY FORCE}

Let's consider an Euler-Bernoulli beam subjected to an arbitrarily given force $P(t)$ moving on the beam with constant speed $v$ as shown in Fig. 1. By introducing the notations $w(x, t)$ for transverse deflection of the beam at section $x$, the governing equation for transverse vibration of the beam can be derived as

$$
E I \frac{\partial^{4} w(x, t)}{\partial x^{4}}+\rho A \eta \frac{\partial w(x, t)}{\partial t}+\rho A \frac{\partial^{2} w(x, t)}{\partial t^{2}}=P(t) \delta(x-v t) .
$$

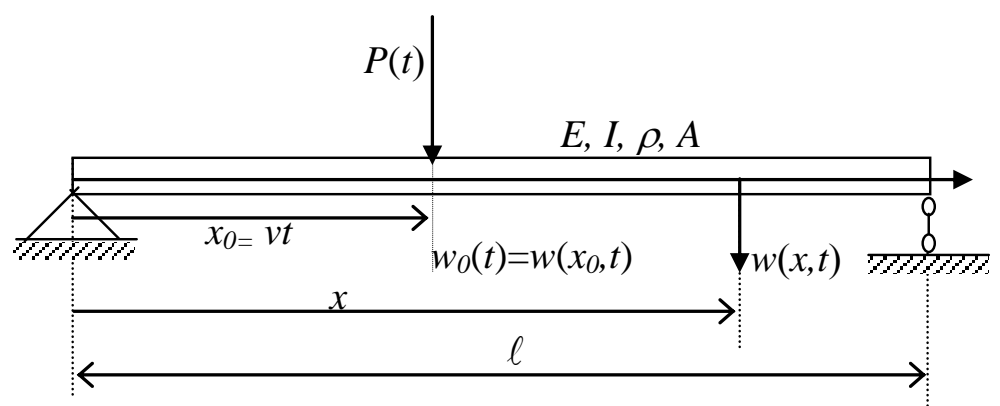

Fig. 1. Dynamic model of beam subjected to moving load

In the latter equation $E, \rho, A, I, \ell$ are material and geometric constants; $\eta$ is damping coefficient of beam and $\delta(t)$ is Dirac delta function. Furthermore, solution of Eq. (1) is subject to general boundary conditions that can be expressed as

$$
\begin{aligned}
& w^{\left(p_{0}\right)}(0, t) \equiv \frac{\partial^{p_{0}} w(0, t)}{\partial x^{p_{0}}}=0, \quad w^{\left(q_{0}\right)}(0, t) \equiv \frac{\partial^{q_{0}} w(0, t)}{\partial x^{q_{0}}}=0, \\
& w^{\left(p_{1}\right)}(\ell, t) \equiv \frac{\partial^{p_{1}} w(0, t)}{\partial x^{p_{1}}}=0, \quad w^{\left(q_{1}\right)}(\ell, t) \equiv \frac{\partial^{q_{1}} w(0, t)}{\partial x^{q_{1}}}=0,
\end{aligned}
$$

where the derivative's orders $p_{0}, q_{0}, p_{1}, q_{1}$ may be equal to one of the values $0,1,2,3$ in dependence on the specific boundary conditions. For example, $p_{0}=p_{1}=0, q_{0}=q_{1}=2$ if the beam is simply supported and $p_{0}=p_{1}=0, q_{0}=q_{1}=1$ when the beam ends are clamped.

The Fourier transform

$$
\phi(x, \omega)=\int_{-\infty}^{\infty} w(x, t) e^{-i \omega t} d t
$$


leads Eq. (1), (2) to

$$
\begin{gathered}
\frac{d^{4} \phi(x, \omega)}{d x^{4}}-\lambda^{4} \phi(x, \omega)=Q(x, \omega), \\
\lambda^{4}=\left(\omega^{2}-i \eta \omega\right) / a^{2}, \quad a=\sqrt{E I / \rho A}, \quad Q(x, \omega)=P(x / v) e^{-i \omega x / v} / E I v, \\
\left.\phi^{\left(p_{0}\right)}(0, \omega) \equiv \frac{d^{p_{0}} \phi}{d x^{p_{0}}}\right|_{x=0}=0,\left.\quad \phi^{\left(q_{0}\right)}(0, \omega) \equiv \frac{d^{q_{0}} \phi}{d x q_{0}}\right|_{x=0}=0, \\
\left.\phi^{\left(p_{1}\right)}(\ell, \omega) \equiv \frac{d^{p_{1}} \phi}{d x^{p_{1}}}\right|_{x=\ell}=0,\left.\quad \phi^{\left(q_{1}\right)}(\ell, \omega) \equiv \frac{d^{q_{1}} \phi}{d x^{q_{1}}}\right|_{x=\ell}=0 .
\end{gathered}
$$

Solution of Eq. (3) satisfying boundary conditions (5) is acknowledged as frequency response of the beam subjected to moving load $P(t)$. It is well known that general solution of Eq. (3) can be represented as

$$
\phi(x, \omega)=\phi_{0}(x, \omega)+\phi_{1}(x, \omega),
$$

where $\phi_{0}(x, \omega)$ is general solution of the homogeneous equation

$$
d^{4} \phi_{0}(x, \omega) / d x^{4}-\lambda^{4} \phi_{0}(x, \omega)=0,
$$

and $\phi_{1}(x, \omega)$ - a particular solution of Eq. (3). The particular solution $\phi_{1}(x, \omega)$ is

$$
\phi_{1}(x, \omega)=\int_{0}^{x} h(x-s) Q(s, \omega) d s, \quad h(x)=(\sinh \lambda x-\sin \lambda x) / 2 \lambda^{3},
$$

that satisfies the conditions

$$
\phi_{1}(0, \omega)=\phi_{1}^{\prime}(0, \omega)=\phi_{1}^{\prime \prime}(0, \omega)=\phi_{1}^{\prime \prime \prime}(0, \omega)=0 .
$$

On the other hand, solution of Eq. (7) can be represented as

$$
\phi_{0}(x)=C L_{1}(x)+D L_{2}(x),
$$

with constants $C, D$ and $L_{1}(x), L_{2}(x)$, being independent particular solutions of Eq. (7) satisfying conditions $L_{k}^{\left(p_{0}\right)}(0)=L_{k}^{\left(q_{0}\right)}(0)=0, k=1,2$. Namely, the functions

$$
L_{1}(\lambda x)=\sinh \lambda x, \quad L_{2}(\lambda x)=\sin \lambda x,
$$

for the simple support and

$$
L_{1}(\lambda x)=\cosh \lambda x-\cos \lambda x, \quad L_{2}(\lambda x)=\sinh \lambda x-\sin \lambda x,
$$

for beam with clamped ends. Obviously, such the functions $L_{1}(x), L_{2}(x)$ make the solutions (6) satisfying the boundary conditions at the left end $x=0$. Substituting expression (6) together with (10) into the boundary conditions (5) at the other end one obtains

$$
C L_{1}^{\left(p_{1}\right)}(\lambda \ell)+D L_{2}^{\left(p_{1}\right)}(\lambda \ell)=-\phi_{1}^{\left(p_{1}\right)}(\ell, \omega), \quad C L_{1}^{\left(q_{1}\right)}(\lambda \ell)+D L_{2}^{\left(q_{1}\right)}(\lambda \ell)=-\phi_{1}^{\left(q_{1}\right)}(\ell, \omega) .
$$

The system of equations in (13) is easily solved with respect to constants $C, D$ and in result it gives

$$
C=\frac{\phi_{1}^{\left(q_{1}\right)}(\ell, \omega) L_{2}^{\left(p_{1}\right)}(\lambda \ell)-\phi_{1}^{\left(p_{1}\right)}(\ell, \omega) L_{2}^{\left(q_{1}\right)}(\lambda \ell)}{L_{1}^{\left(p_{1}\right)}(\lambda \ell) L_{2}^{\left(q_{1}\right)}(\lambda \ell)-L_{1}^{\left(q_{1}\right)}(\lambda \ell) L_{2}^{\left(p_{1}\right)}(\lambda \ell)}, D=\frac{\phi_{1}^{\left(p_{1}\right)}(\ell, \omega) L_{1}^{\left(q_{1}\right)}(\lambda \ell)-\phi_{1}^{\left(q_{1}\right)}(\ell, \omega) L_{1}^{\left(p_{1}\right)}(\lambda \ell)}{L_{1}^{\left(p_{1}\right)}(\lambda \ell) L_{2}^{\left(q_{1}\right)}(\lambda \ell)-L_{1}^{\left(q_{1}\right)}(\lambda \ell) L_{2}^{\left(p_{1}\right)}(\lambda \ell)} .
$$


Thus, a closed form solution of Eq. (3) with boundary conditions (5) has been found and it is exact frequency response for deflection of beam subject to moving arbitrary load. Furthermore, the solution allows one to calculate the response for slope, moment and shear force respectively as

$$
\phi^{(r)}(x, \omega)=C L_{1}^{(r)}(\lambda x)+D L_{2}^{(r)}(\lambda x)+\phi_{1}^{(r)}(x, \omega), r=1,2,3 .
$$

It is of interest to consider specially the case of multiple harmonic force

$$
P(t)=\sum_{k=1}^{m} P_{k} e^{i\left(\Omega_{k} t+\theta_{k}\right)},
$$

that includes particularly the constant $\left(\Omega_{k}=0\right)$ and harmonic forces or their combination. In such the case the frequency response can be found in the form

$$
\phi(x, \omega)=\sum_{k=1}^{m} \phi_{k}(x, \omega)
$$

where

$$
\phi_{k}(x, \omega)=C_{k} L_{1}(\lambda x)+D_{k} L_{2}(\lambda x)+\phi_{1 k}(x, \omega) .
$$

The constants $C_{k}, D_{k}$ in (18) are calculated by using (14) with the function

$$
\begin{gathered}
\phi_{1 k}(\lambda x)=Q_{k}\left[A_{1} \cosh \lambda x-A_{2} \sinh \lambda x+A_{3} \cos \lambda x-A_{4} \sin \lambda x-A_{0} e^{-i \hat{\omega} x / v}\right], \\
A_{0}=\frac{1}{\lambda^{4}-(\hat{\omega} / v)^{4}}, \quad A_{1}=\frac{1}{2 \lambda^{2}\left[\lambda^{2}+\left(\hat{\omega}_{k} / v\right)^{2}\right]}, \quad A_{3}=\frac{1}{2 \lambda^{2}\left[\lambda^{2}-\left(\hat{\omega}_{k} / v\right)^{2}\right]}, \\
A_{2}=\frac{\left(i \hat{\omega}_{k} / v\right)}{2 \lambda^{3}\left[\lambda^{2}+\left(\hat{\omega}_{k} / v\right)^{2}\right]}, A_{4}=\frac{\left(i \hat{\omega}_{k} / v\right)}{2 \lambda^{3}\left[\lambda^{2}-\left(\hat{\omega}_{k} / v\right)^{2}\right]}, Q_{k}=P_{k} e^{i \theta_{k}} / E I v, \hat{\omega}_{k}=\omega-\Omega_{k} .
\end{gathered}
$$

The frequency response $\phi(x, \omega)$ found above is a complex function with real and imaginary parts $\phi_{R}(x, \omega), \phi_{I}(x, \omega)$, so that the following spectral characteristics for the response can be defined

$$
S_{w}(x, \omega)=|\phi(x, \omega)|=\left(\phi_{R}^{2}+\phi_{I}^{2}\right)^{1 / 2}, \quad \Phi_{w}(x, \omega)=\tan ^{-1}\left(\phi_{R} / \phi_{I}\right) .
$$

The former function considered as function of frequency $\omega \in\left[\bar{\omega}_{a}, \bar{\omega}_{b}\right]$ for a fixed $x_{0}$ is termed by amplitude spectrum of the dynamic response at the span location $x_{0}$. That characteristic considered as function of span location $x \in[0, L]$ for particular frequency $\omega_{0}$ is called herein amplitude diagram (vibration mode) at the frequency. The latter function in (21) represents phase evolution of response in the frequency domain. In dynamic analysis of structure subjected to moving harmonic force the following frequencies are specially emphasized: natural frequencies of structure denoted by $\omega_{k}, k=1,2,3, \ldots$, among that the fundamental one $\omega_{1}$ is most important; driving frequency $\omega_{v}=\pi v / \ell$ governed by the load speed and, finally, load frequency $\Omega$, the frequency of the moving harmonic force. The vibration components of response at the natural, driving and load frequencies are called respectively natural, driven and forced mode of total dynamic response. It has to note that amplitude of natural mode calculated at fundamental frequency is not identical to amplitude of the first mode examined in the modal superposition method. 


\section{NUMERICAL RESULTS AND DISCUSSION}

In this section, a simply supported Euler-Bernoulli beam of the parameters: $\ell=$ $30 \mathrm{~m} ; E I=1.42 E 10 \mathrm{Nm}^{2} ; \rho A=4800 \mathrm{~kg} / \mathrm{m}$ subjected to moving force $P(t)=P_{0}\left[\alpha e^{i \Omega_{1} t}+\right.$ $\left.\beta e^{i \Omega_{2} t}\right]$ is examined. Though the harmonic force including the constant one as its particular case $(\Omega=0)$ was intensively studied in the time domain, some essential aspects of the moving load can be straightforwardly revealed in the spectral point of view. Hence, frequency response to both the single constant and harmonic forces is investigated herein by the spectral approach before analysis of the multiple harmonic force case. For convenience, the following dimensionless parameters are introduced: speed factor $\gamma=v / v_{c}=\omega_{v} / \omega_{1}$; load frequency ratio $f_{e}=\Omega / \omega_{1}$ and spectrum is examined versus dimensionless frequency $\bar{\omega}=\omega / \omega_{1} \in[0,2]$.

\subsection{Frequency response to constant force}

Amplitude spectrums of mid-span deflection for various load speeds are given in Fig. 2 that allows one to identify vibration components of response with different frequencies in dependence on the speed. Namely, in the low speed range $(\gamma \prec 0.1)$ the deflection spectrum can be interpreted by the function

$$
S_{v}(\omega)=\frac{S_{0} \cos \left(\pi \omega / 2 \omega_{v}\right)}{\left|\omega_{v}^{2}-\omega^{2}\right|}
$$

that is actually calculated from the sinusoidal impulse

$$
q_{v}(t)= \begin{cases}a_{0} \sin \omega_{v} t & \text { if } t \in[0, T] \\ 0 & \text { otherwise }\end{cases}
$$

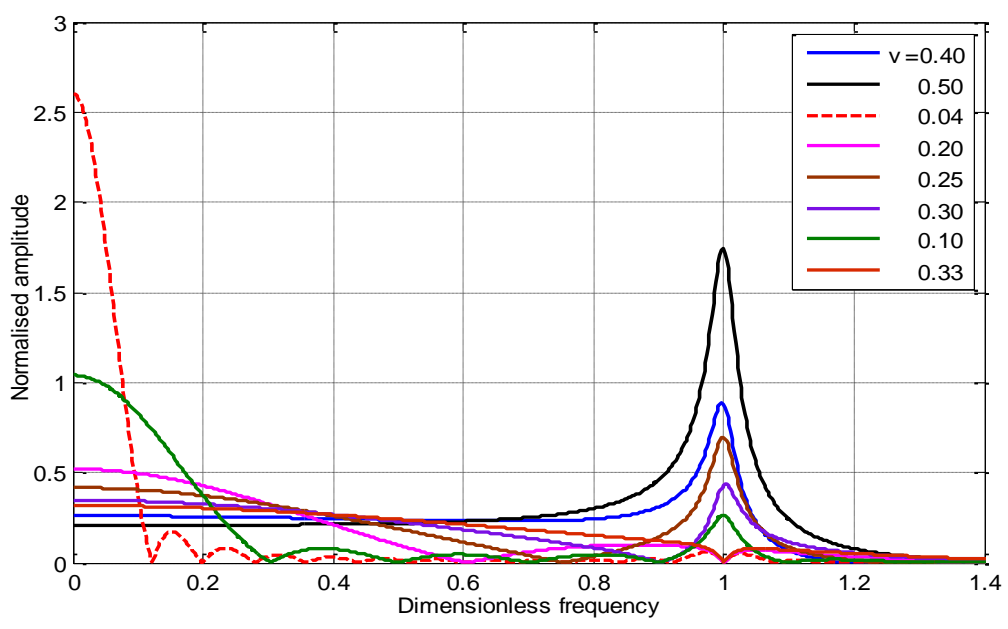

Fig. 2. Spectrum for midspan deflection response to moving constant force with various speeds

In the above equations $\omega_{v}=\pi v / \ell=\pi / T ; T=\ell / v ; a_{0}=S_{0} \omega_{v} / 2$ where $S_{0}=$ $\phi(\ell / 2,0)$ is the defection at $\omega=0$. The function (23) is an important component of the 
time history response with driving frequency $\left(\omega_{v}=\pi v / \ell\right)$ that is termed above by the driven vibration mode. Comparison of the spectrum (23) to the exact one given in Fig. 2 for the speed factor equal to 0.05 and 0.09 is shown in Fig. 3. The excellent agreement of the spectrums demonstrates domination of the driven vibration mode in the low speed range. The spectrum (23) normalized by the factor $S_{0} / 2 \omega_{v}^{2}$ gives exactly the dynamic amplification coefficient provided in [17].

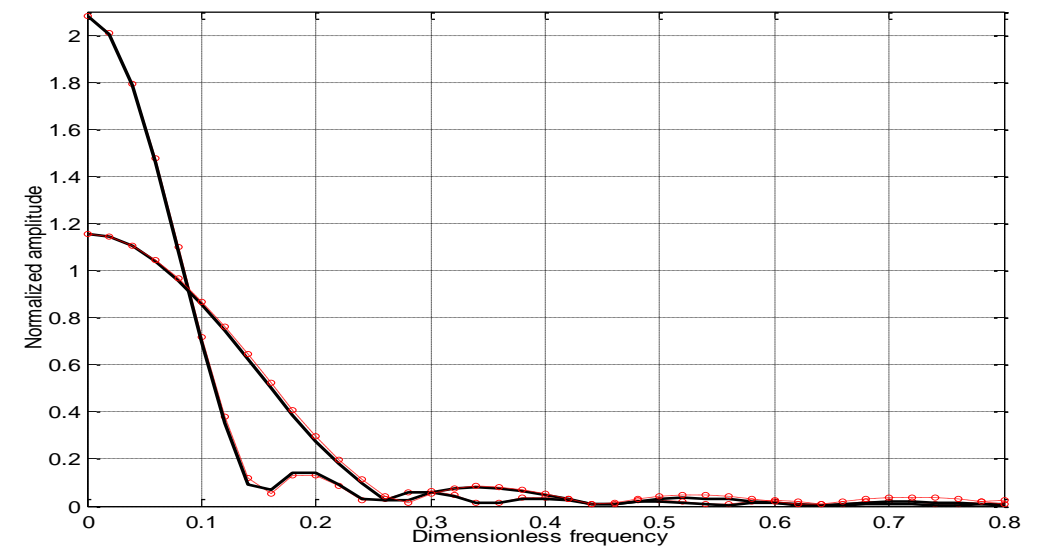

Fig. 3. Comparison of the exact and approximate spectrums: solid line - exact; dash line - approximate

It can be observed on Fig. 2 that in the high speed range $(\gamma \succ 0.35)$ the spectrum of response is similar to that of a single degree of freedom oscillator (only one peak at the natural frequency). This implies that response component of natural frequency is predominating for high speed. In the medium range of speed $(0.1 \leq \gamma \leq 0.35)$ the driven and natural modes are strongly coupled so that it can be observed cancellation of response amplitude at natural frequency for several speeds (0.04, 0.2 and 0.33). Fig. 4(a) displays amplitudes of natural vibration mode as function of speed factor for various damping ratios that demonstrate more clearly the cancellation of response at natural frequency. Comparing the graphs given in Fig. 4(a) with amplitude calculated by Eq. (7), Ref. [24] allows one to note that the latter equation obtained as the first mode approximation of response gives accurate solution with damping ratio equal 0.0265 .

Amplitude of response at driving frequency in dependence on speed and damping is given in Fig. 4(b) that shows interesting fact that amplitude of driven mode response is almost independent on damping until the speed factor approaching 0.8 . It is rapidly dropping with speed factor up to 0.3 and remains nearly constant for speed factor ranged in the interval $(0.3-0.8)$. For the speed factor ranged in $(0.8-1.0)$ the amplitude becomes increasing with speed and decreasing with growing damping likely the natural vibration mode at resonance.

Since amplitude of alone natural vibration mode of response reaches its maximum almost at natural frequency, total response amplitude at the frequency could be canceled 


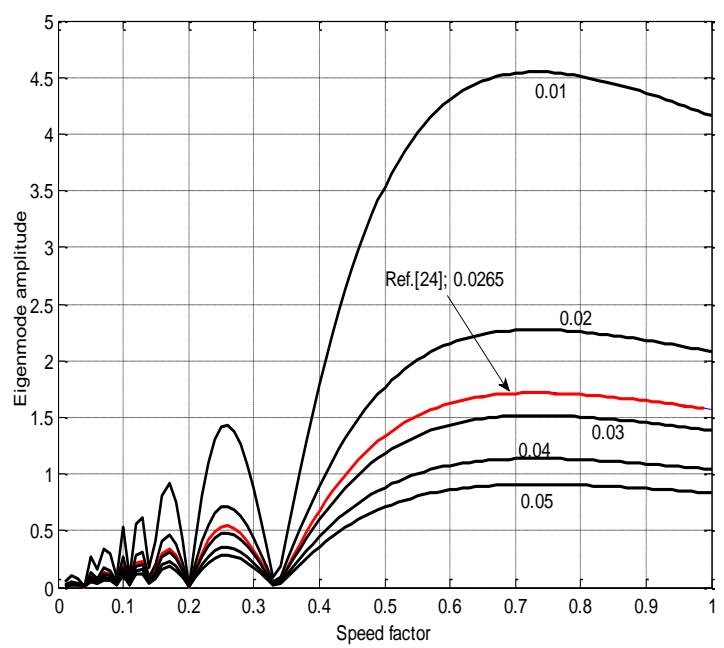

(a)

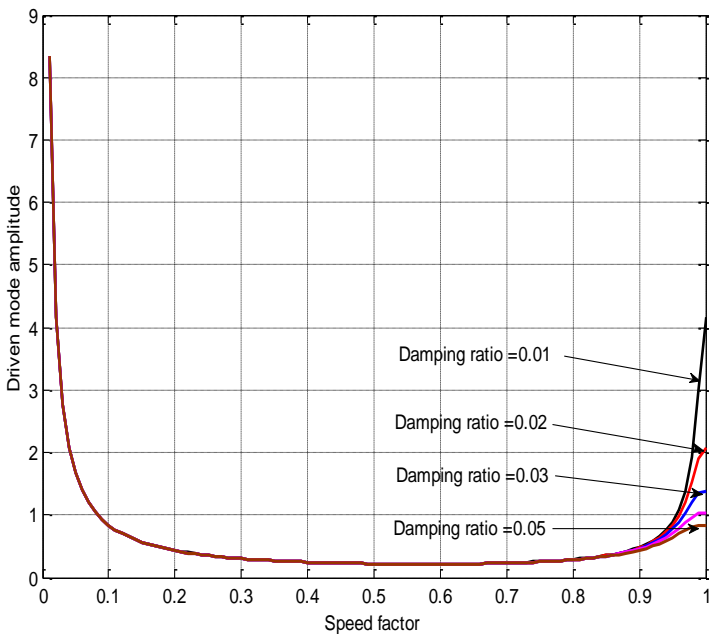

(b)

Fig. 4. Amplitude of eigenmode (a) and driven mode (b) response under constant force

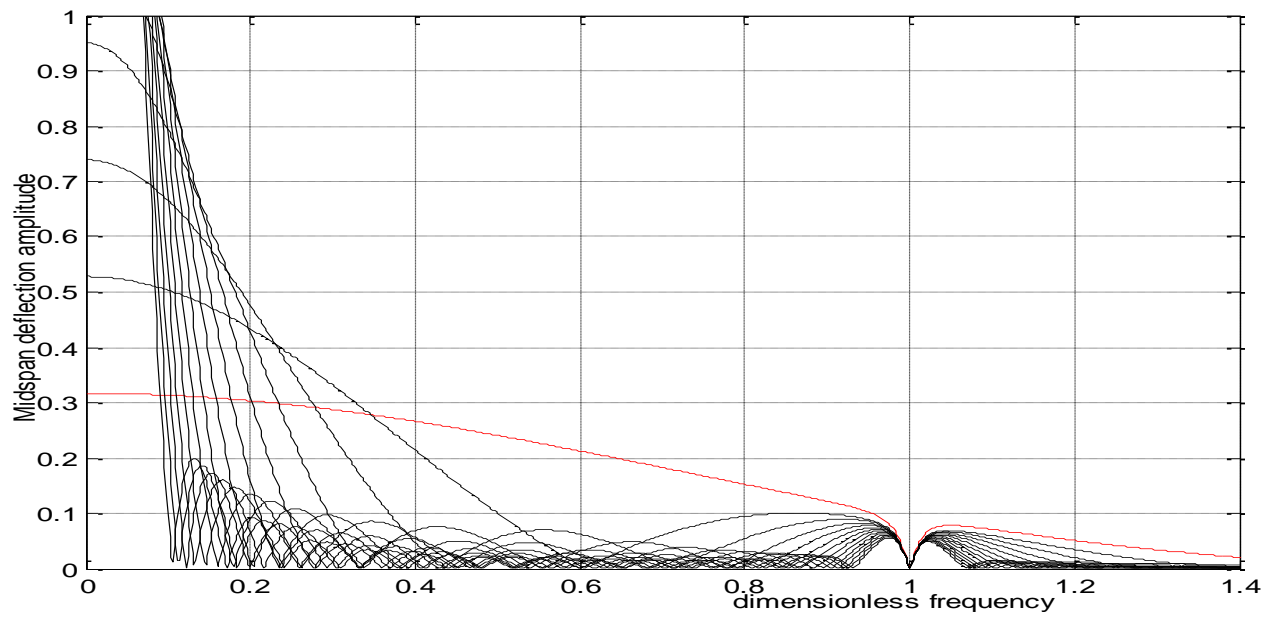

Fig. 5. Response amplitude spectrum at antiresonant speeds - Suppression of the eigenmode response

only if amplitude of driven mode of response vanishes, i. e.

$$
\cos \pi \omega_{1} / 2 \omega_{v}=0 \quad \text { or } \quad \omega_{1}=(2 k+1) \omega_{v}, \quad k=1,2,3, \ldots
$$

From the latter equation so-called cancellation speeds can be calculated $\gamma_{k}=1 /(2 k$ $+1), k=1,2,3, \ldots$ called in this study anti-resonant speeds. These speeds have been found in [22] by using the superposition method in time domain. The response spectrums calculated for the exact anti-resonant speeds are presented in Fig. 5 that validate actual annulation of amplitude of response at natural frequency. 


\subsection{Frequency response to harmonic force}

Amplitude spectrum of normalized midpoint deflection in the case $\Omega=0.4 \omega_{1}$ is shown in Fig. 6 for various speeds. It can be seen that forced vibration mode is dominated for speed factor less than 0.1 (Fig. 6(a)). However, the peak at the frequency is rapidly decreasing and completely disappeared when speed factor approaches to 0.2. For the speed factor higher than 0.2 there is observed only one peak at natural frequency that implies predomination of vibration mode of response at natural frequency in this speed range. The driven vibration mode of response appears as small waves in both sides of the forced vibration peak. Likely to the case of constant load, the anti-resonant speeds can be found from the equation

$$
\cos \left[\left(\omega_{1}-\Omega\right) \pi / 2 \omega_{v}\right]=0,
$$

that gives rise anti-resonant speed factor

$$
\gamma_{e k}=\left|1-f_{e}\right| /(2 k+1), k=1,2,3, \ldots
$$

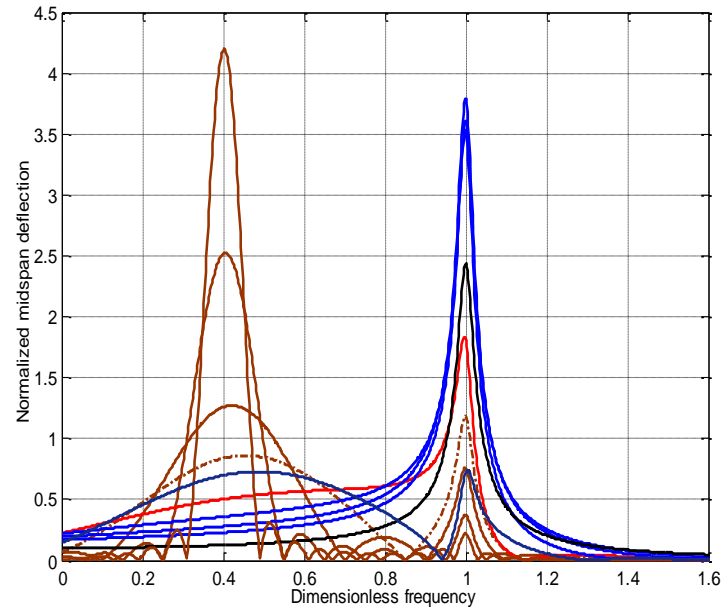

(a) Not anti-resonant speed

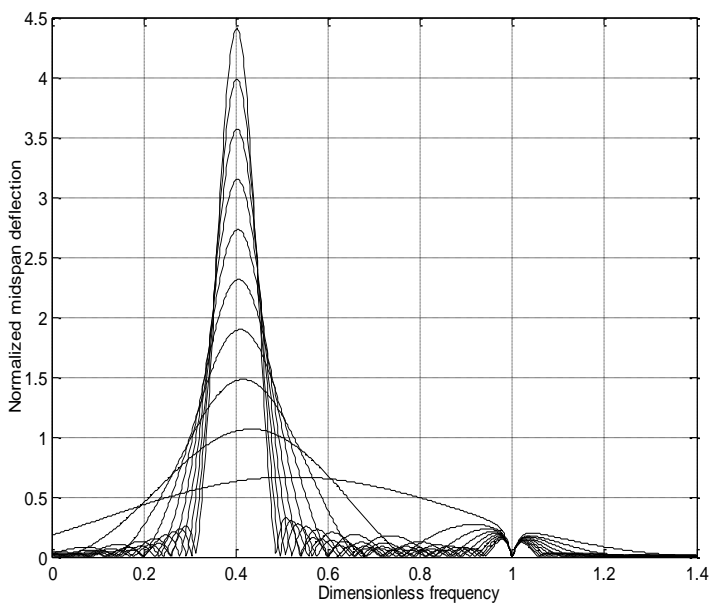

(b) Anti-resonant speeds

Fig. 6. Midpoint deflection spectrum for various speed and load frequency $\Omega=0.4 \omega_{1}$

The cancellation of natural mode response at anti-resonant speeds (26) is obviously verified by graphs of the spectrums (Fig. 6(b)) calculated for the speeds. Dependence of the anti-resonant speed on frequency of moving force for different integer number $k$ is interpreted in Fig. 7. In the case when moving force frequency coincides with driving one, i. e. $\gamma_{e}=f_{e}$, referred to as external resonance, the Eq. (26) yields

$$
\gamma_{\text {erk }}=1 / 2(k+1), \quad k=1,2,3, \ldots
$$

The natural mode amplitude at external resonance in dependence on speed is provided in Fig. 8 from that it can be observed global maximum of eigenmode amplitude reached at speed equal a half of critical value $\left(0.5 V_{c}\right)$. Fig. 9 shows amplitude of forced 
vibration mode (a) and natural mode response (b) plotted versus speed with different frequency of moving force. Graphs given in Fig. 9(a) demonstrate monotonic decrease of the forced vibration mode of response with increasing speed. The natural mode vibration amplitudes shown in Fig. 9(b) reach their maximum at the speeds varying with the moving force frequency. The maximum speeds are tabulated in Tab. 1 for load frequencies from 0 to $0.9 \omega_{1}$.

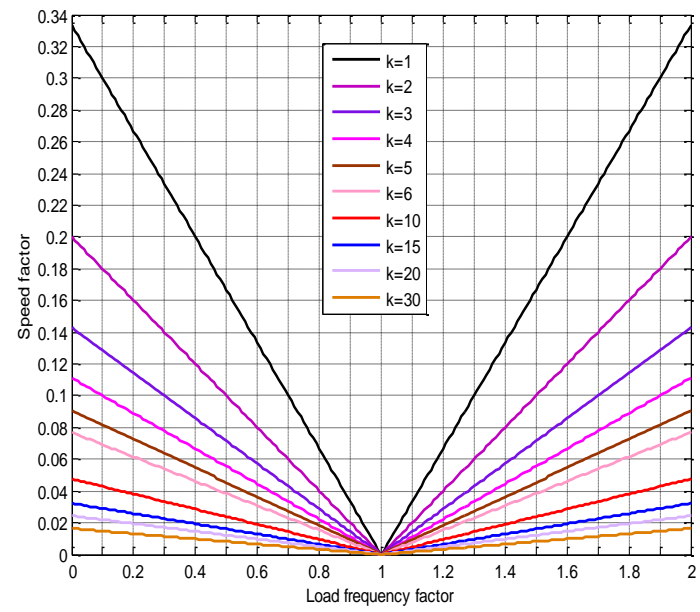

Fig. 7. Speed-Load frequency map at the suppression of eigenmode response to single harmonic force

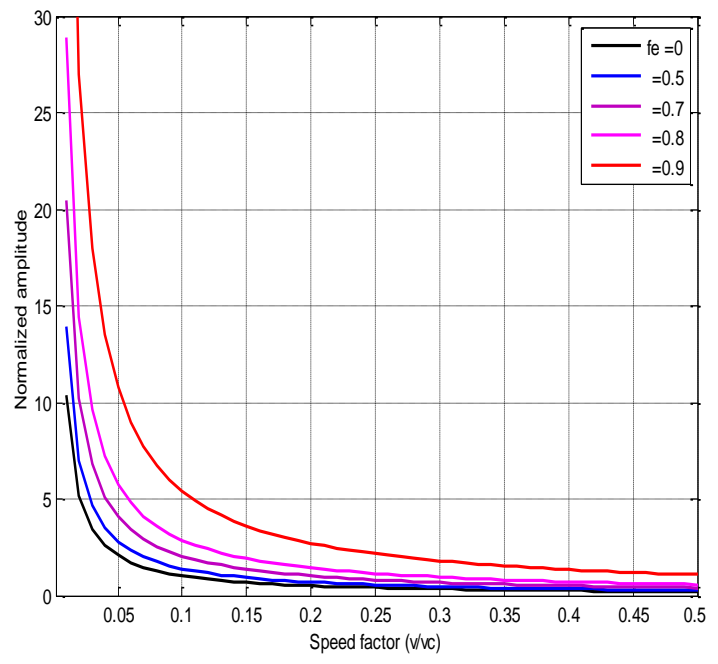

(a)

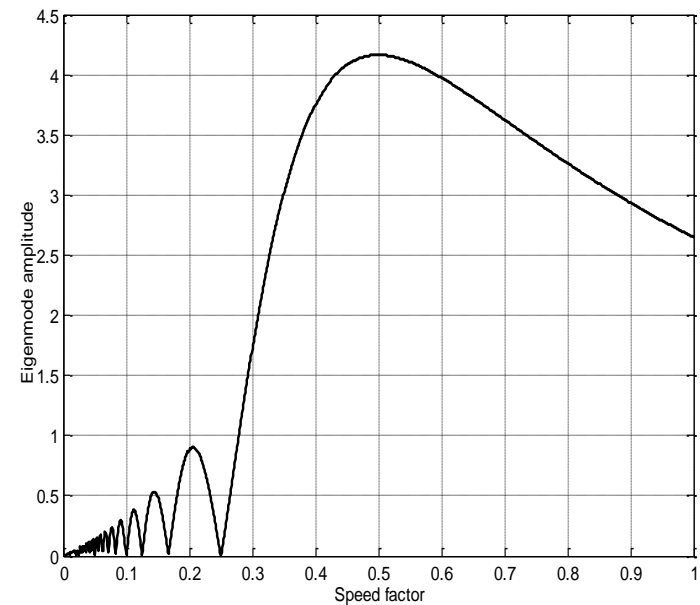

Fig. 8. Eigenmode amplitude at the external resonance (equality of load and driving frequency

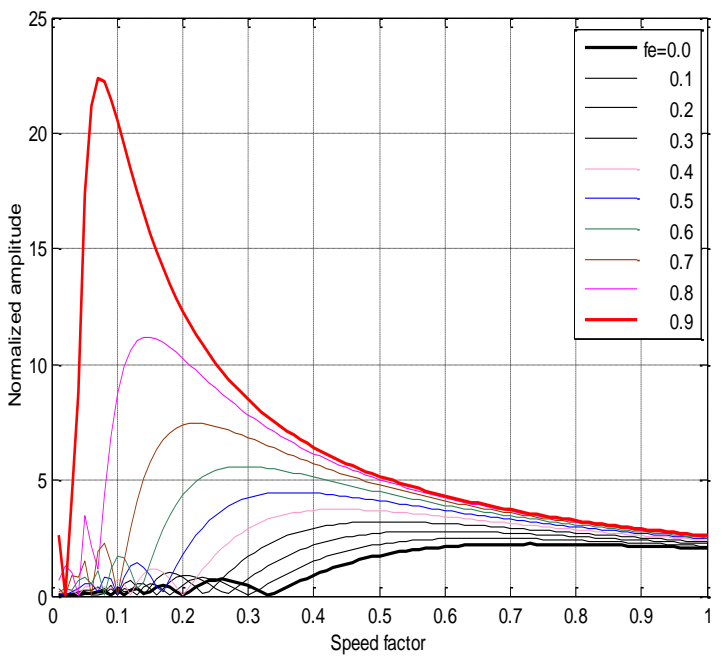

(b)

Fig. 9. Amplitude of forced (a) and natural mode (b) versus speed factor 
Table 1. Speed for maximum amplitude of response in different frequency modes

\begin{tabular}{|c|c|c|c|c|c|c|c|c|c|}
\hline \multicolumn{10}{|c|}{ Load frequency factor } \\
\hline 0 & 0.1 & 0.2 & 0.3 & 0.4 & 0.5 & 0.6 & 0.7 & 0.8 & 0.9 \\
\hline \multicolumn{8}{|c|}{ Speed factor for maximum natural mode amplitude } \\
\hline 0.731 & 0.658 & 0.585 & 0.512 & 0.439 & 0.366 & 0.293 & 0.219 & 0.146 & 0.073 \\
\hline
\end{tabular}

Amplitudes of the driven vibration mode of response versus speed factor for different frequency of moving force are plotted in Fig. 10. Obviously, the driven vibration amplitude first increases to a local maximum and then decreases to a minimum with speed factor rising up to 0.6. Afterward, the amplitude gets to increase with speed approaching the critical value. At the critical speed the driven (but not natural mode) response attains its global maximum that increases with the load frequency.

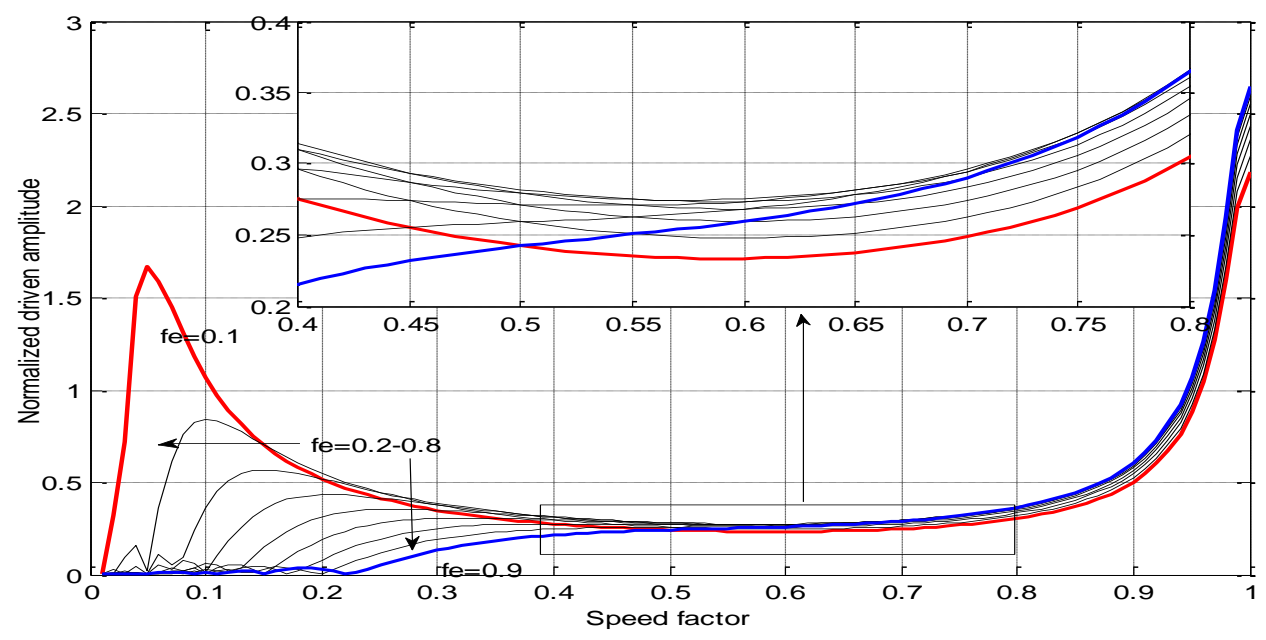

Fig. 10. Amplitude of driven response versus speed factor and load frequency

\subsection{Effect of combined harmonic forces}

Note first that harmonic forces with symmetrical frequencies $\Omega_{1}, \Omega_{2}$, such that $\left(\Omega_{1}+\Omega_{2}\right) / 2=\omega_{1}$, individually produce the same effect on the natural mode amplitude (dash lines in Fig. 11). Consequently, anti-resonant speeds corresponding to the symmetric harmonic forces are identical and the harmonic force with super resonant frequency $\Omega=2 \omega_{1}$ has the same effect on the eigenmode amplitude as the constant force. However, combined excitation of symmetrical harmonic forces (solid lines, Fig. 11) has not only amplified global maximum amplitude of the natural mode response but also generated new antiresonant speed. Namely, it is evident from Fig. 11 that the global maximum is higher about 1.7 times than that produced by individual harmonic force of equal magnitude. Besides, the common antiresonant speeds of the symmetric harmonic loads 
calculated by Eq. (27) with $k=1,2,3, \ldots$ are added by a new one that is calculated from the same equation with $k=0$. Therefore, the speed $v=\left|1-f_{e}\right| V_{c}$ is also antiresonant for the combined symmetric harmonic loads and, as consequence, the critical speed $V_{c}$ becomes anti-resonant for the load combined from constant $\Omega_{1}=0$ and super resonant $\Omega_{2}=2 \omega_{1}$ forces.

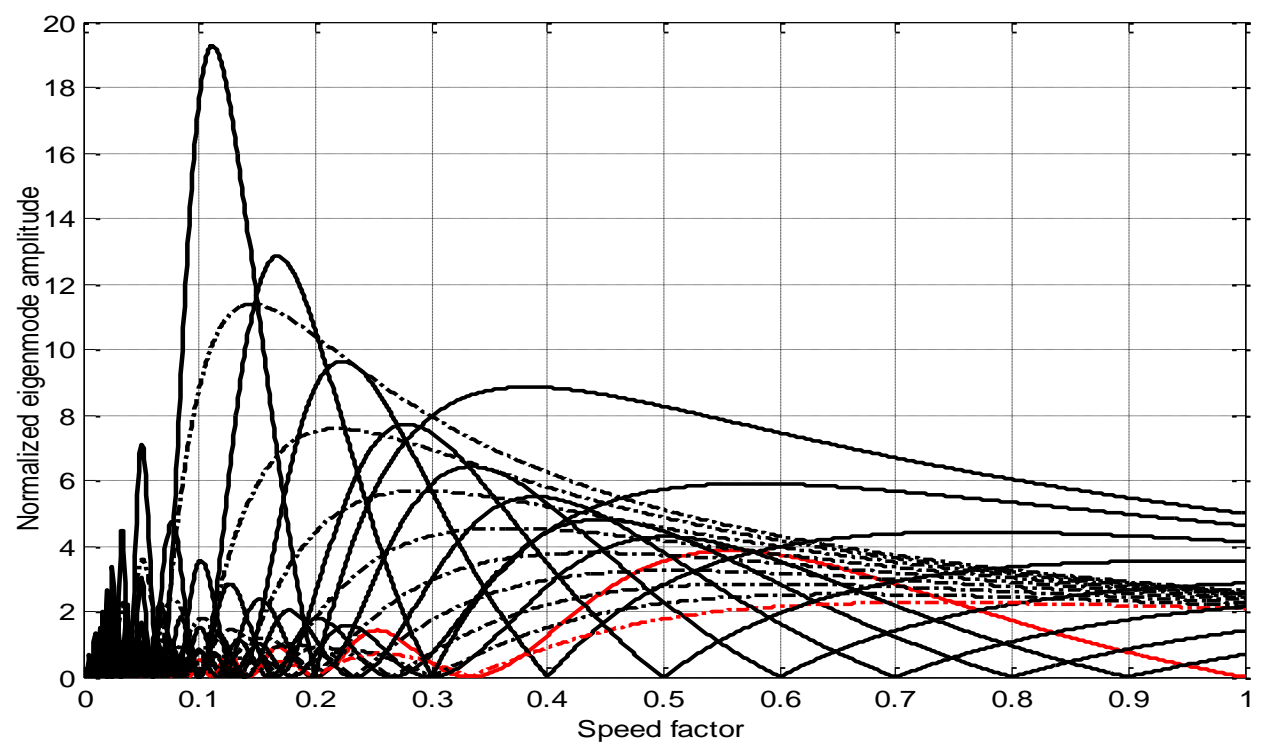

Fig. 11. Natural mode amplitude of response under combined symmetric harmonic loads

The typical combination of non-symmetric harmonic loads is the case of joined constant and harmonic forces. Amplitude of natural mode response subject to the combined force is examined for the following cases of excitation frequency: (1) exceeded by fundamental frequency $\left(\Omega_{1}=0 \prec \Omega_{2} \prec \omega_{1}\right)$ and (2) exceeding the natural frequency $\left(\Omega_{1}=0 \prec \omega_{1} \prec \Omega_{2}\right)$ and results are presented in Fig. 12. For comparison, the natural mode amplitudes of response caused by alone harmonic load of frequency $\left(0 \prec \Omega \prec 2 \omega_{1}\right)$ are given also in Fig. 12 (solid lines). It can be observed from the figure that effect of constant load on the natural mode response to combined load (shortly, combined response) in both the cases of load frequency is small for speed factor less than 0.5 .

However, the effect becomes more significant for speed factor exceeding 0.5. Namely, in comparison with response amplitude caused by single harmonic force, the combined force with frequency higher (lower) the fundamental frequency produces greater (smaller) the response amplitude. Therefore, a conclusion can be made that contribution of constant force to the combined response is dependent on whether the force frequency is higher or lower the fundamental frequency. The constant force makes no effect on the natural mode amplitude of combined response at its anti-resonant speeds, but effect of harmonic load gets to be more significant as its frequency approaches to the resonant one. 


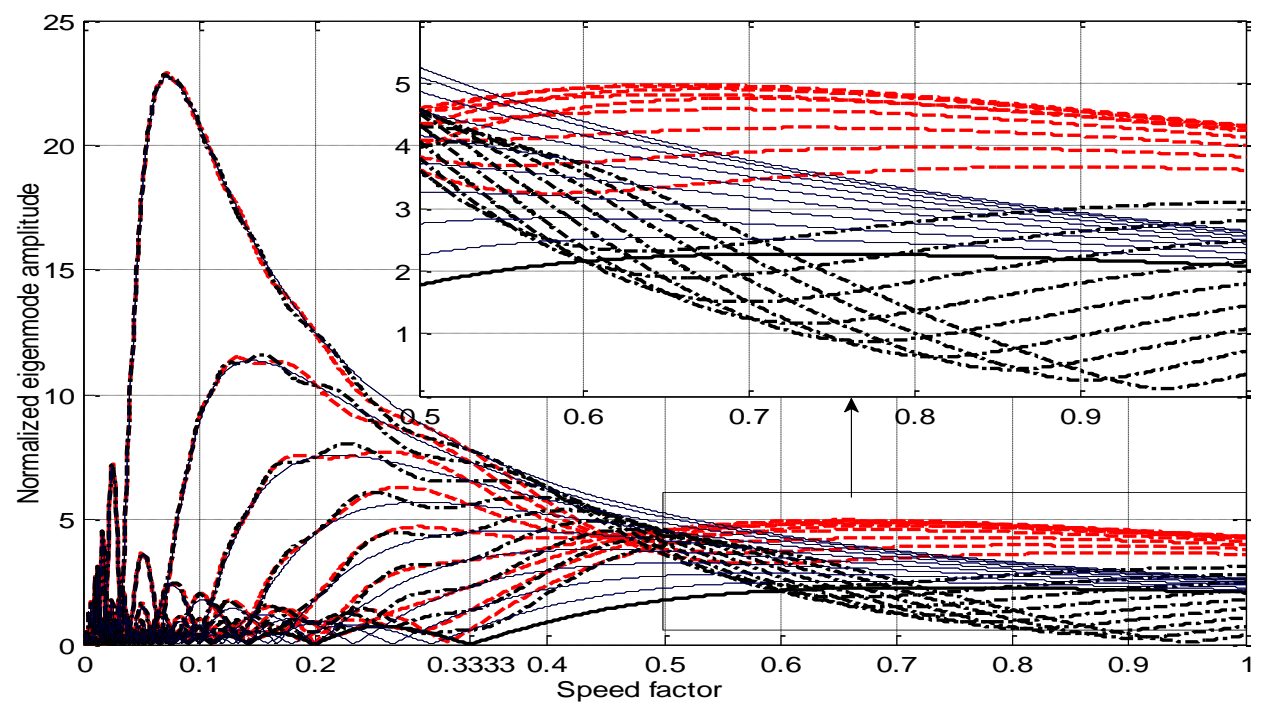

Fig. 12. Natural mode amplitude of response under combined constant and harmonic loads

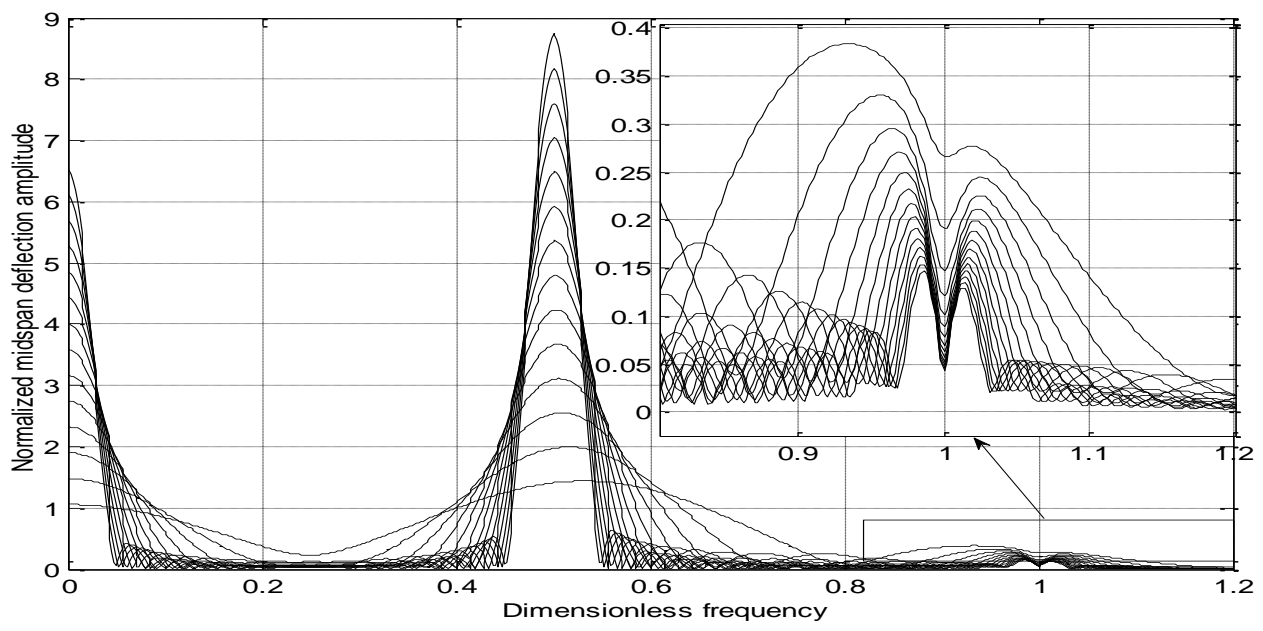

Fig. 13. Response spectrum under combined constant $(\Omega=0)$ and harmonic $\left(\Omega=0.5 \omega_{1}\right)$ forces for anti-resonant speeds

Natural mode response cannot be completely canceled by combination of non-symmetric harmonic forces but it may be reduced to minimum (Fig. 13).

\section{CONCLUSION}

Summarizing the main results obtained in present study the following conclusions can be made: 
1. An analytical expression for frequency response of beam-like structure subjected to moving arbitrary force is obtained as an exact solution of the moving load problem in the frequency domain. The arbitrary force model includes wide variety of loading such as the constant, harmonic or random forces and their combinations. It may be also used for studying dynamic response of structure to successive harmonic loads.

2. Using the obtained solution the dynamic response of a simply supported beam under multiple harmonic loads travelling with constant speed is thoroughly investigated in the frequency domain. This spectral analysis shows to be more simple and consistent tool for characterizing the majored frequency components of response (called hereby natural mode, driven and forced modes correspondingly to natural, driving and loading frequencies respectively) and their interaction causing different meanings of resonance.

3. Namely, the forced mode response dominates for the lower speed and it attains maximum at the traditional resonance, but its amplitude decreases monotonically and rapidly with growing speed. The driven mode response appears as numerous small petals in the response spectrum and achieves its global maximum under the critical speed (when driving frequency equal natural one). Both the response components, in contrary to the natural mode, are slightly dependent on the damping. Natural mode response is really leading for high speed of force travelling and, as usually, reaches its maximum amplitude at resonance. Moreover, the spectral approach provides an alternative insight to the cancellation of natural mode response that allows calculating so-called anti-resonant speeds for individual and combined harmonic loads.

4. It was revealed that action of individual harmonic load is strongly dependent on the distance between natural frequency and load frequency. Namely, the harmonic loads with frequencies symmetrical in both side of a natural frequency individually create the same effect on amplitude of natural mode response. This means in particular that constant load and harmonic load with super resonant frequency $\left(\Omega=2 \omega_{1}\right)$ do the same effect on the natural mode response. However, resultant effect of combined harmonic loads with non-symmetrical frequencies is leaded sturdily by the load that has frequency more closed to resonant.

5. Dynamic response of a beam subjected to a moving force usually achieves maximum amplitude at the mid-span except the case when speed of the load roving equals to the anti-resonant speed. This allows one to make a conclusion that the first mode approximation in the superposition method applied for the moving load problem is consistent only in the case if load speed is not anti-resonant.

6. Influence of magnitude and phase of the harmonic loads shows to be actually monotonic so that is not specified herein. However, the phase of moving load would take an important role in the case of successive moving loads that is a further subject of study for the authors. Finally, the spectral approach developed above and illustrated for simply supported beam can be easily applied for investigating the beams with other boundary conditions.

\section{REFERENCES}

[1] L. Frỳba. Vibration of solids and structures under moving loads. Groningen: The Netherland, (1972). doi:10.1007/978-94-011-9685-7. 
[2] M. Olsson. On the fundamental moving load problem. Journal of Sound and Vibration, 145, (2), (1991), pp. 299-307. doi:10.1016/0022-460x(91)90593-9.

[3] J. Li and M. Su. The resonant vibration for a simply supported girder bridge under high-speed trains. Journal of Sound and Vibration, 224, (5), (1999), pp. 897-915. doi:10.1006/jsvi.1999.2226.

[4] Y. K. Cheung, F. T. K. Au, D. Y. Zheng, and Y. S. Cheng. Vibration of multi-span non-uniform bridges under moving vehicles and trains by using modified beam vibration functions. Journal of Sound and Vibration, 228, (3), (1999), pp. 611-628. doi:10.1006/jsvi.1999.2423.

[5] G. V. Rao. Linear dynamics of an elastic beam under moving loads. Journal of Vibration and Acoustics, 122, (3), (2000), pp. 281-289. doi:10.1115/1.1303822.

[6] M. Klasztorny and J. Langer. Dynamic response of single-span beam bridges to a series of moving loads. Earthquake Engineering \& Structural Dynamics, 19, (8), (1990), pp. 1107-1124. doi:10.1002/eqe.4290190803.

[7] M. A. Hilal and H. S. Zibdeh. Vibration analysis of beams with general boundary conditions traversed by a moving force. Journal of Sound and Vibration, 229, (2), (2000), pp. 377-388. doi:10.1006/jsvi.1999.2491.

[8] A. V. Pesterev, B. Yang, L. A. Bergman, and C. A. Tan. Response of elastic continuum carrying multiple moving oscillators. Journal of engineering mechanics, 127, (3), (2001), pp. 260-265. doi:10.1061/(asce)0733-9399(2001)127:3(260).

[9] C. Bilello and L. A. Bergman. Vibration of damaged beams under a moving mass: theory and experimental validation. Journal of Sound and Vibration, 274, (3), (2004), pp. 567-582. doi:10.1016/j.jsv.2003.01.001.

[10] H. P. Lin and S. C. Chang. Forced responses of cracked cantilever beams subjected to a concentrated moving load. International Journal of Mechanical Sciences, 48, (12), (2006), pp. 14561463. doi:10.1016/j.ijmecsci.2006.06.014.

[11] R. Zarfam and A. R. Khaloo. Vibration control of beams on elastic foundation under a moving vehicle and random lateral excitations. Journal of Sound and Vibration, 331, (6), (2012), pp. 1217-1232. doi:10.1016/j.jsv.2011.11.001.

[12] Y. H. Lin and M. W. Trethewey. Finite element analysis of elastic beams subjected to moving dynamic loads. Journal of Sound and Vibration, 136, (2), (1990), pp. 323-342. doi:10.1016/0022460x(90)90860-3.

[13] J. J. Wu, A. R. Whittaker, and M. P. Cartmell. The use of finite element techniques for calculating the dynamic response of structures to moving loads. Computers $\mathcal{E}$ Structures, 78, (6), (2000), pp. 789-799. doi:10.1016/s0045-7949(00)00055-9.

[14] K. Henchi, M. Fafard, G. Dhatt, and M. Talbot. Dynamic behaviour of multi-span beams under moving loads. Journal of Sound and Vibration, 199, (1), (1997), pp. 33-50. doi:10.1006/jsvi.1996.0628.

[15] N. Azizi, M. M. Saadatpour, and M. Mahzoon. Using spectral element method for analyzing continuous beams and bridges subjected to a moving load. Applied Mathematical Modelling, 36, (8), (2012), pp. 3580-3592. doi:10.1016/j.apm.2011.10.019.

[16] N. T. Khiem, T. H. Tran, and N. V. Quang. An approach to the moving load problem for multiple cracked beam. In Proceeding of the 31st IMAC, A Conference on Structural Dynamics, USA, 2013: Topics in Modal Analysis, Vol. 7. Springer, (2013), pp. 451-460. doi:10.1007/978-14614-6585-0_43.

[17] E. Savin. Dynamic amplification factor and response spectrum for the evaluation of vibrations of beams under successive moving loads. Journal of Sound and Vibration, 248, (2), (2001), pp. 267-288. doi:10.1006/jsvi.2001.3787. 
[18] A. V. Pesterev, B. Yang, L. A. Bergman, and C. A. Tan. Revisiting the moving force problem. Journal of Sound and Vibration, 261, (1), (2003), pp. 75-91. doi:10.1016/s0022-460x(02)00942-2.

[19] Y. B. Yang and C. W. Lin. Vehicle-bridge interaction dynamics and potential applications. Journal of Sound and Vibration, 284, (1), (2005), pp. 205-226. doi:10.1016/j.jsv.2004.06.032.

[20] A. Garinei. Vibrations of simple beam-like modelled bridge under harmonic moving loads. International Journal of Engineering Science, 44, (11), (2006), pp. 778-787. doi:10.1016/j.ijengsci.2006.04.013.

[21] F. Ricciardelli and C. Briatico. Transient response of supported beams to moving forces with sinusoidal time variation. Journal of Engineering Mechanics, 137, (6), (2010), pp. 422-430. doi:10.1061/(asce)em.1943-7889.0000241.

[22] Y. B. Yang, J. D. Yau, and L. C. Hsu. Vibration of simple beams due to trains moving at high speeds. Engineering Structures, 19, (11), (1997), pp. 936-944. doi:10.1016/s0141-0296(97)000011.

[23] Y. B. Yang, C. L. Lin, J. D. Yau, and D. W. Chang. Mechanism of resonance and cancellation for train-induced vibrations on bridges with elastic bearings. Journal of Sound and Vibration, 269, (1), (2004), pp. 345-360. doi:10.1016/s0022-460x(03)00123-8.

[24] P. Museros, E. Moliner, and M. D. Martínez-Rodrigo. Free vibrations of simply-supported beam bridges under moving loads: Maximum resonance, cancellation and resonant vertical acceleration. Journal of Sound and Vibration, 332, (2), (2013), pp. 326-345. doi:10.1016/j.jsv.2012.08.008.

[25] C. Sudheesh Kumar, C. Sujatha, and K. Shankar. Vibration of non-prismatic simply supported beams under moving loads: cancellation of resonances. In Proceedings of 11th International Conference on Vibration Problems, Lisbon, Portugal, (2013). pp. 1-10. 